\title{
Die Rolle des Timings am BeIspiel ZEITGENÖSSISCHER JAZZGITARRISTEN*
}

\author{
Márton Szegedi
}

Wenngleich die Forschungsliteratur zur Mikrorhythmik im Jazz in den letzten zwei Jahrzehnten beständig zugenommen hat, bleibt ihr Umfang im Vergleich zu harmonie- bzw. melodiebezogenen Untersuchungen verhältnismäßig gering. Dies ist in erster Linie wohl dem Sachverhalt zuzuschreiben, dass die melodische bzw. harmonische Sprache des Jazz im Wesentlichen der europäisch-klassischen Musiktradition entspringt und diese wiederum althergebrachte Analyseinstrumentarien zur Verfügung stellt. Eben solche Instrumentarien existieren hinsichtlich feinrhythmischer jedoch Gestaltung kaum. Die ersatzweise Verwendung gewisser Termini (zu denen auch der Begriff Timing zählt) deckt zwar die Grundbedürfnisse kommunikativen Austausches ab, bleibt aber - da in der Regel nie hinterfragt - inhaltlich nebulös.

Im Rahmen der vorliegenden Arbeit wird der Versuch unternommen, die meistverwendeten Timingformen am Beispiel der wichtigsten Protagonisten der zeitgenössischen Jazzgitarrenszene darzustellen. Nach der Schilderung wesentlicher theoretischer Grundlagen des Timings werden verschiedene Aspekte diverser gängiger Timingstrukturen anhand ausgewählter Interpretationsanalysen von John Scofield, Pat Metheny, Mike Stern, Bill Frisell sowie Jesse van Ruller untersucht. Diese Vorgehensweise ist musikimmanent geleitet und hebt sich derart bewusst vom Großteil der einschlägigen Forschungsliteratur $a b$, welche primär naturwissenschaftlichen Untersuchungsmethoden folgt. ${ }^{1}$ Diese Forschungsliteratur aus einem musikalisch-spielästhetischen Blickwinkel zu komplettieren ist das größere Ziel der vorliegenden Untersuchung. Ihr Ausgangspunkt ist die klingende Musik, die wesentliche Methode die elaborierte Höranalyse. Deren Ergebnisse mög-

* Dieses Forschungsprojekt wurde gefördert vom Austrian Science Fund (FWF): P 23453-G21.

1 Vgl. Clarke 1999; Honing 2001; Kvifte 2007; Marsden 2007; Goebl/Palmer 2009; Butterfield 2010. 
lichst sichtbar und kommunizierbar zu machen dienen die eigens erstellten Transkriptionen, wie sie auch die besondere Möglichkeit eröffnen, die Denkweise des Interpreten nachzuvollziehen.

Vor allem im Bereich der mündlich oder auditiv überlieferten Musikformen, wie Jazz oder Volksmusik, gibt es zwischen notiertem und gespieltem Rhythmus kleinere wie auch größere Abweichungen. ${ }^{2}$ Diese werden im Wesentlichen durch antizipierendes bzw. retardierendes Spiel erzeugt. Unter Timing ist mithin das Spannungsfeld zwischen (konventionell) notiertem und realisiertem Rhythmus zu verstehen.

Dieses Phänomen verfügt über zwei grundlegende Eigenschaften, deren erste die Ausrichtung ist. Damit ist gemeint, dass ein Rhythmus entweder (1) exakt, der Notation möglichst genau entsprechend, oder (2) später bzw. (3) früher als notiert - oder als erwartet - ausgeführt wird. Aus den letzteren zwei Möglichkeiten ergibt sich die zweite Charakteristik: das Ausmaß. Die realisierten Rhythmen können von mikrorhythmischen bis hin zu makrorhythmischen, exzessiven Modifikationen reichen.

\begin{tabular}{|c|c|}
\hline Ausrichtung $\quad \longrightarrow$ & Ausmaß \\
\hline $\left.\begin{array}{ll}\text { 1. } & \text { exakt } \\
\text { 2. } & \text { retardierend } \\
\text { 3. } & \text { antizipierend }\end{array}\right\}$ & $\begin{array}{l}\text { 1. mikrorhythmisch } \\
\text { 2. exzessiv }\end{array}$ \\
\hline
\end{tabular}

Tabelle 1: Eigenschaften des Timings

All dies wirft die Frage auf, welche musikalischen Basisfaktoren den Gebrauch unterschiedlicher Timingtypen bedingen. Um dieser Frage nachgehen zu können, wurden für die nachfolgenden Interpretationsanalysen die Schwerpunkte »exaktes Timing «, »Swing « und »bimetrische Modelle« ausgewählt, ergänzt um eine ansatzweise Darstellung diverser weiterer Strukturen. Diese Kategorien lassen sich aus dem Gesamtinventar der mehr als 60 Transkriptionen des Autors herleiten und werden im Weiteren anhand jeweils eines markanten Beispiels verdeutlicht. Als rhythmischer Bezugspunkt dient jeweils das von der Rhythmusgruppe vorgegebene, kontinuierlich gleich bleibende Metrum.

2 Unter Notation sind zunächst Lead Sheets, Big Band-Partituren sowie herkömmliche, rhythmisch vereinfacht aufgezeichnete Transkriptionen zu verstehen. 


\section{Exaktes Timing}

Beim exakten Timing handelt es sich um »metronomisch korrektes « Spiel, wobei auf jegliche mikro- bzw. makrorhythmische Modifikation verzichtet wird. Falls Töne ab und zu retardierend bzw. antizipierend ausgeführt sind (Pfeile in den Transkriptionen), mag es der unvermeidbaren menschlichen Ungenauigkeit zugeschrieben werden.

Im untersuchten Sample kommt exaktes Timing am häufigsten in Stücken vor, denen eine binäre Beat-Unterteilung zugrunde liegt. Dazu gehören einerseits die auf divisiver Rhythmik basierenden, von der Rockmusik beeinflussten Richtungen, wie das nachfolgende Fusion-Stück:

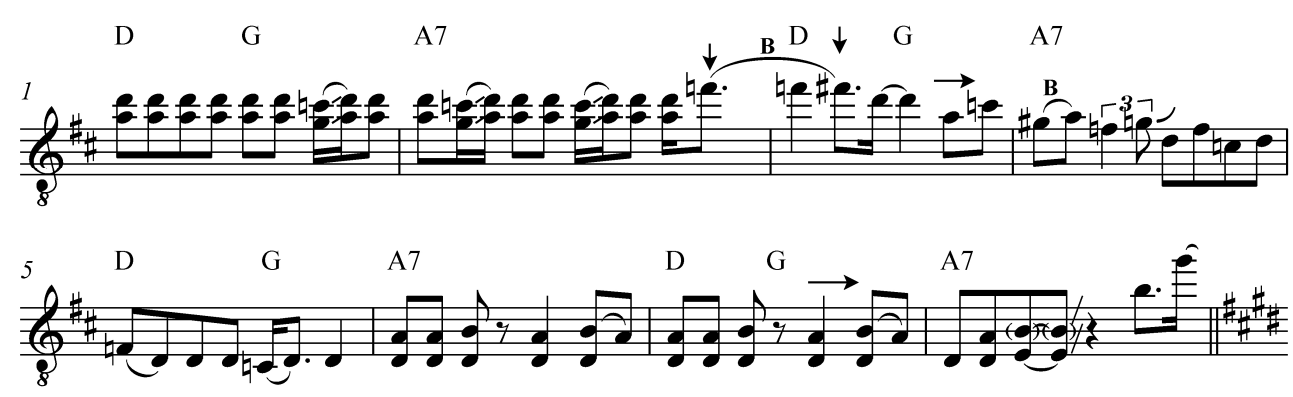

Abb. 1: Bill Frisell - »Twister«, Improvisationschorus / B (binär) ${ }^{3}$

Andererseits gibt es die auf Clave-Patterns beruhenden Latin-Stücke mit additiver Rhythmik, wie im unteren Beispiel des holländischen Gitarristen Jesse van Ruller, in dessen Komposition »Secret Champ« die mit Akzent versehenen Diskant-Bordun-Töne fast immer mit den Impakten des Bossa NovaRhythmus zusammenfallen, was eines genauen Platzierens der Töne bedarf:

3 In den Transkriptionen finden folgende diakritische Zeichen sowie Symbole Verwendung:

- nach rechts weisender Pfeil - mikrorhythmisches Retardieren,

- nach links weisender Pfeil - mikrorhythmisches Antizipieren,

- »B« - Bending,

- Strich zwischen Notenköpfen - chromatisches Glissando,

- nach unten weisender Pfeil - tiefer als notiert,

- nach oben weisender Pfeil - höher als notiert,

- x-förmiger Notenkopf - kaum hörbarer Ton,

- rhombischer Notenkopf - Flageolettton,

- kursive Akkordsymbole - Grundakkorde ohne Tensions,

- nach oben weisender Bogen neben dem Notenkopf - mikrorhythmisches, durch Bending erzeugtes Portamento aufwärts,

- nach unten weisender Bogen neben dem Notenkopf - mikrorhythmisches, durch Bending erzeugtes Portamento abwärts,

- Achtelnotenpaar ohne Notenköpfe, über den Noten platziert - binäre Stelle über ternärer Beatunterteilung. 


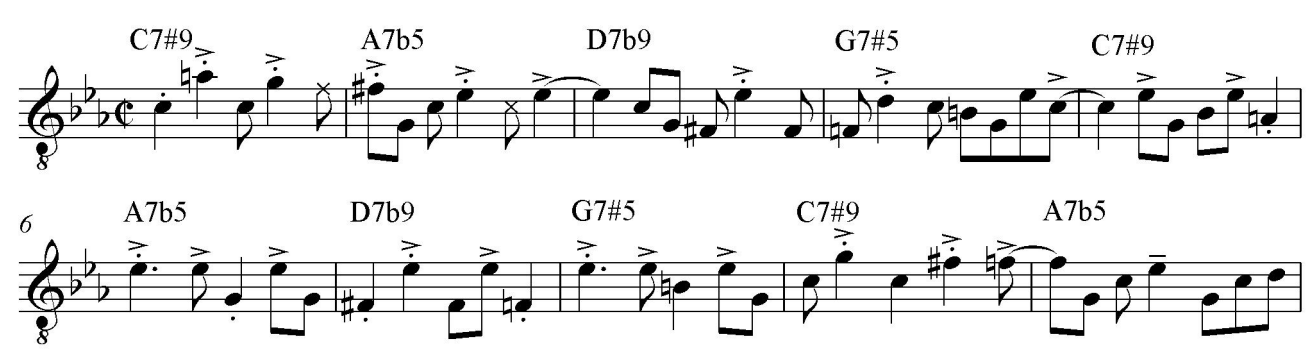

Abb. 2: Jesse van Ruller - »Secret Champ«, Thema / T. 1-10 (binär)

Darüber hinaus lassen sich sehr langsame ternäre Interpretationen zum Großteil ebenfalls unter diese Kategorie subsumieren:

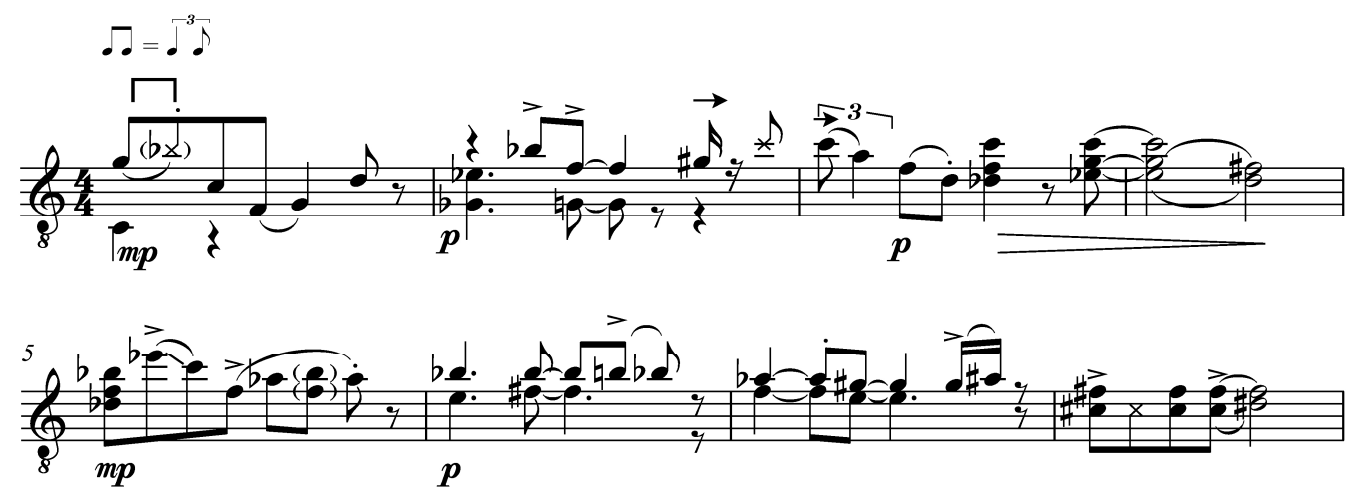

Abb. 3: John Scofield - »Nocturnal Mission«, Thema 2 / T. 1-4 (ternär)

\section{Swing}

Zu einem der wichtigsten rhythmischen Phänomene des Jazz gehört die so genannte Swingphrasierung, welche im Solospiel hauptsächlich dadurch realisiert wird, dass die jeweils zweiten Glieder einer Achtelkette innerhalb einer ternären Interpretation etwas zu früh gespielt werden: ${ }^{4}$

4 Dieser aus musikimmenenter Sicht schlüssigen Definition stehen diverse, in den Ergebnissen zum Teil erstaunlich divergierende Untersuchungen gegenüber. Vgl. generell Parsons/Cholakis 1995, Collier/Collier 1996 und Honing/Haas 2008 zur Ride-Cymbal-Figur; Prögler 1995 zum Zusammenspiel von Schlagzeug und Bass; Friberg/Sundström 2002 zum Zusammenspiel von Schlagzeug und Solisten; Ellis 1991, Busse 2002, Collier/Collier 2002 und Benadon 2006 zum melodischen Solospiel. 
triolische Notation

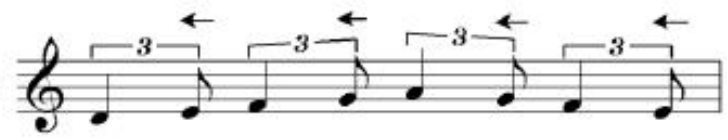

ternäre Notation

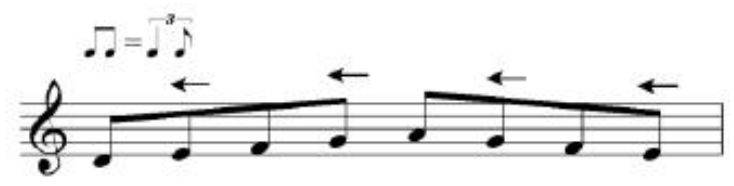

Abb. 4: Swingphrasierung

Das Ausmaß dieser Abweichung lässt sich allerdings nicht leicht ausmachen: Wird der Beat als eine Linie visualisiert, beginnt der erste Ton - da er rhythmisch exakt realisiert wird - bei $0 \%$. Im Falle eines binären Modells fällt der zweite Ton auf 50\%, was der Relation 1:1 entspricht. Betrachtet man die exakte ternäre Realisierung, befindet sich der zweite Ton bei 66,6\%, mit der Proportion 2:1. Falls der zweite Ton im Sinne der Swingphrasierung verzögert wird, sollte er sich irgendwo zwischen diesen beiden Eckpunkten befinden:

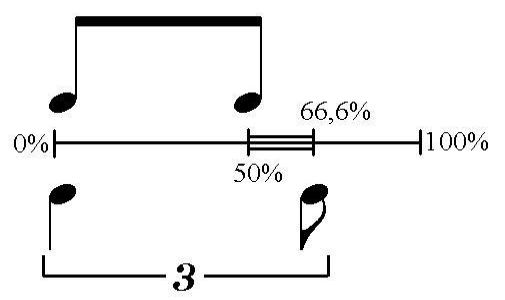

Abbildung 5: Swing

Durch eingehende Untersuchung mittels Messung der Abweichungen ${ }^{5}$ stellt sich allerdings heraus, dass auf jeder Zählzeit eine andere mikrorhythmische Ausführung stattfindet - Musiker sind eben keine Maschinen. Somit ist eine bloß approximative Darstellung auf theoretischer Ebene durchaus gerechtfertigt. ${ }^{6}$ Gleichwohl ist festzustellen, dass das Maß dieser Abweichung größtenteils vom Tempo abhängt. Je schneller nämlich ein Stück ist, desto mehr tendiert die Ausführung in Richtung binärer Phrasierung.

5 Diesbezügliche Literatur s. Fußnote 4.

6 Folgender Sachverhalt bietet sich aufgrund seiner mathematischen Schlüssigkeit für weitere Untersuchungen an: Der Mittelwert zwischen gerader und triolischer Realisierung ist bei 58,3\% anzusiedeln und durch die Proportion 7:5 gekennzeichnet. Wenn aber angenommen wird, dass das zweite Glied näher zum ternären, also zu $66,6 \%$, als zum binären Punkt steht, kann dies am einfachsten mit den Zahlen der Fibonacci-Reihe beschrieben werden: $3: 2$, 5:3 sowie 8:5. Genauso wie die Folge der Quotienten aufeinanderfolgender Fibonacci-Zahlen gegen den Goldenen Schnitt konvergiert, wird der zweite Ton annähernd an jenem Teilungspunkt platziert. 
Dagegen werden ganz langsame Swing-Stücke zumeist beinahe triolisch exakt ausgeführt (vgl. Abbildung 3). Bei extrem schnellen Stücken ist die Abweichung von der ternären Beat-Unterteilung so stark, dass die Achtellinien überwiegend binär klingen:

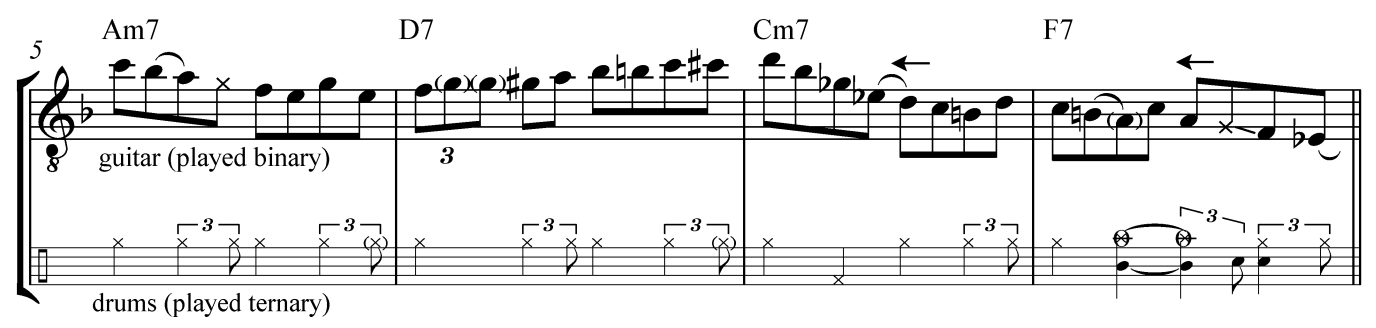

Abb. 6: Mike Stern - »Outta Town«, Improvisationschorus 1 / A2 / T. 5-8 (ternär)

Manchmal kommt eine swingähnliche Phrasierung auch in binären Stücken vor, was sich auch in der Notation darstellen lässt. Das Denkmodell wird demnach umgekehrt:

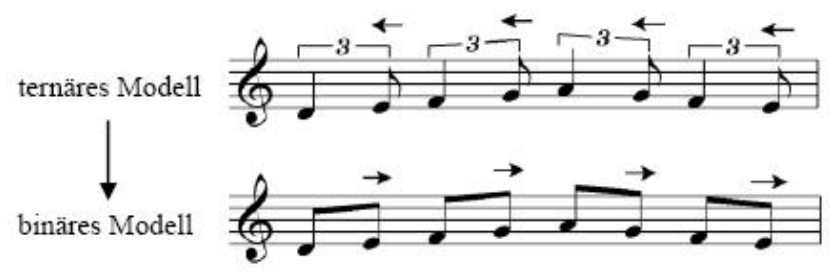

Abb. 7: Binärer Swing

Im folgenden Beispielstück spielen Saxophon und Gitarre unisono anhand des zuvor geschilderten Modells:

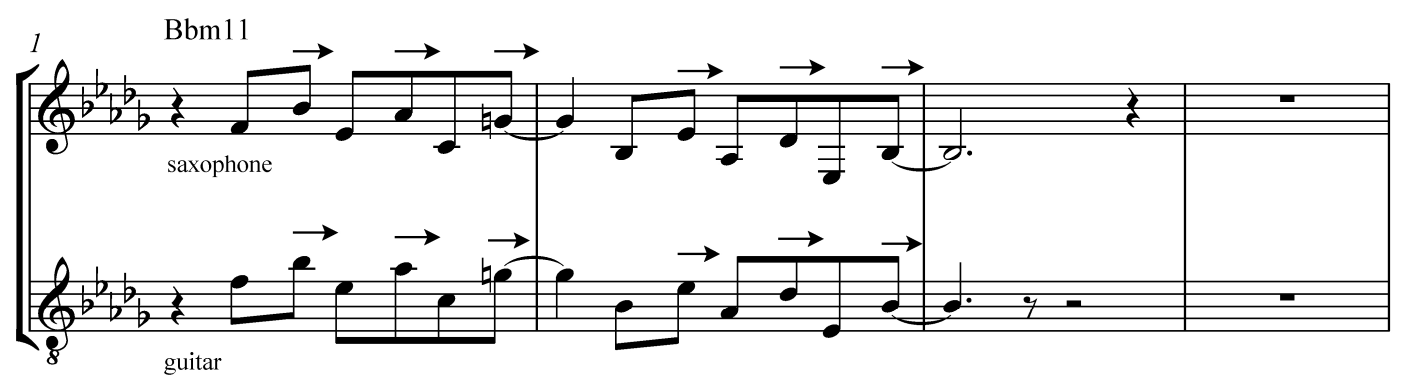

Abb. 8: Joe Lovano (sax) \& John Scofield (g) - »|'ll Catch You«, Thema / A / T. 1-4 (binär)

Im mittleren Tempo fungiert die Swingphrasierung als Ausgangspunkt einer weiteren Art des Timings, bei der gesamte Phrasen antizipiert bzw. retardiert ausgeführt werden: 


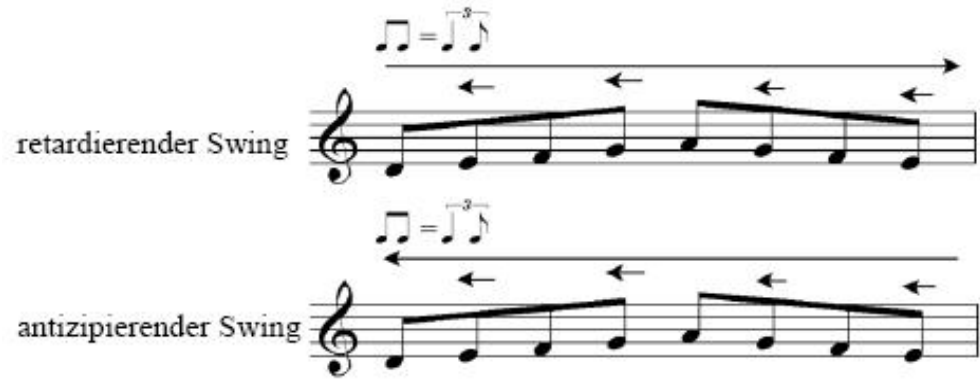

Abb. 9: Antizipierender bzw. retardierender Swing

Dabei ist die antizipierende Gestaltung nicht sehr typisch. Bei den untersuchten Interpretationen lässt sich diese kaum beobachten. Umso häufiger findet man dagegen die retardierende Swingphrasierung vor. Hierbei kann die Notation zur Gänze umgedeutet werden. So kommt der jeweils auf den Beat fallende Ton einer Achtellinie etwas zu spät:

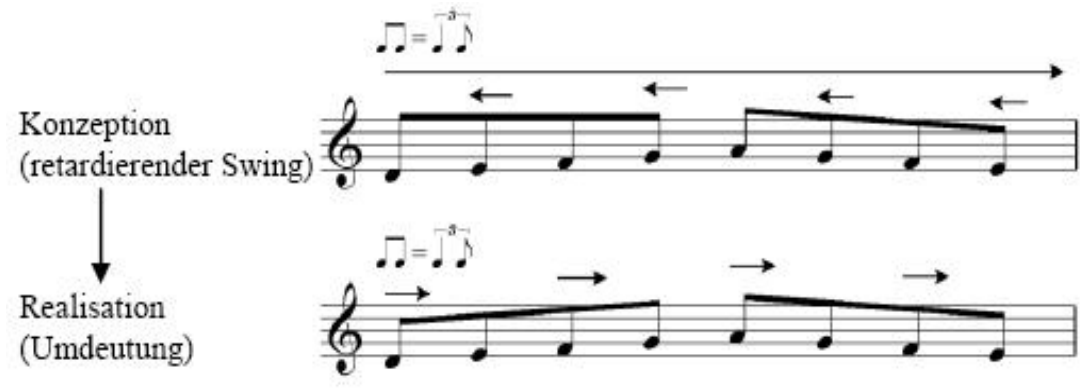

Abb. 10: Retardierender Swing

John Scofield bedient sich in Swing-Stücken mittleren Tempos zumeist dieses Schemas:

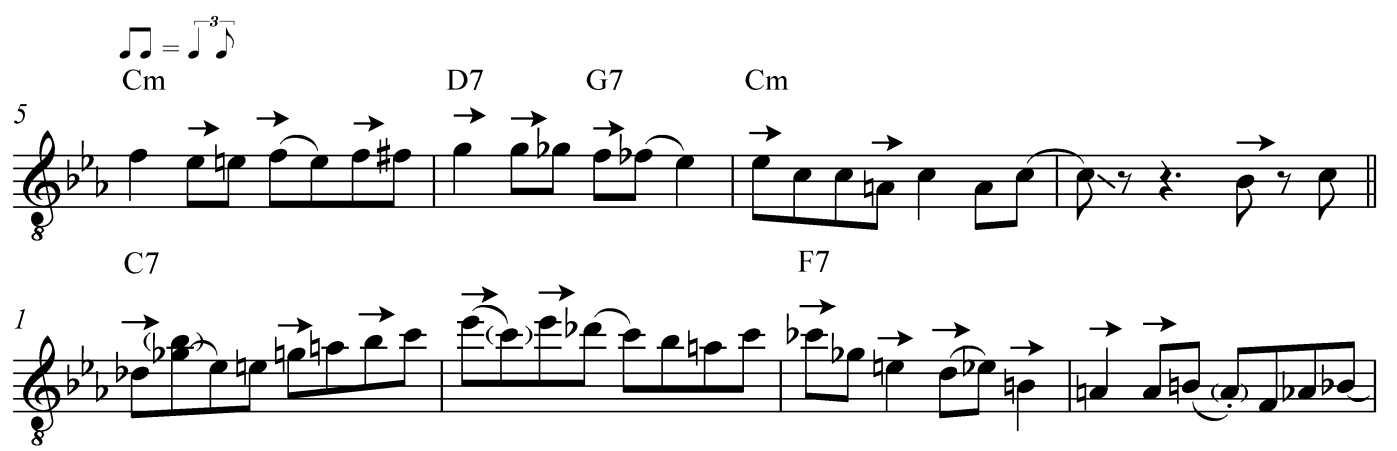

Abb. 11: John Scofield - »Dickie's Dream«, Impr. / A2 / T. 5 - B / T. 3 (ternär)

In langsameren Interpretationen wird diese Gestaltungsweise insofern markanter, als der auf den Beat fallende Ton genau um eine Sechzehnteltriole später ausgeführt wird: 


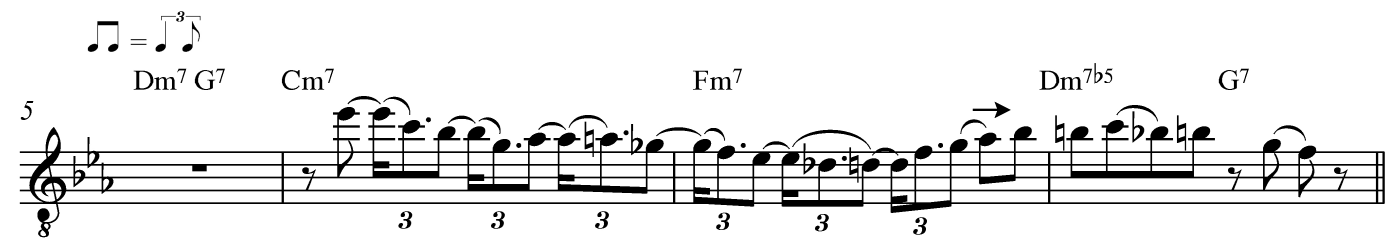

Abb. 12: John Scofield - »I Found A New Baby«, Impr. 1 / B / T. 5-8 (ternär)

Dadurch entspricht die Tonfolge einer binären Realisation, denn wir haben innerhalb eines Beats zwei verschobene, jedoch gleich lange Töne. Es entsteht mithin eine polymetrische Struktur, indem über eine ternäre Begleitung zur gleichen Zeit eine quasi binäre Melodierhythmik ausgeführt wird:

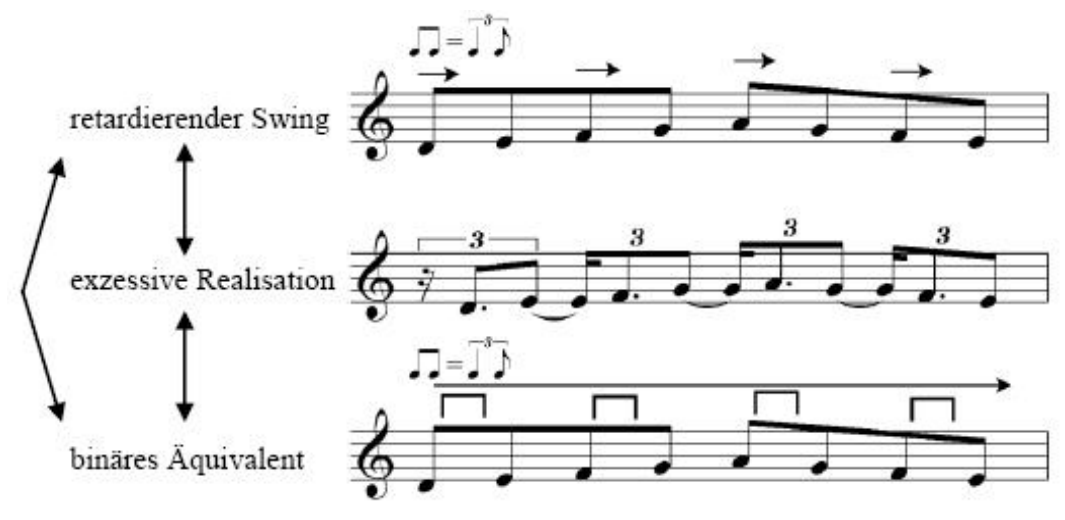

Abb. 13: Exzessiver Swing

Dieses Modell fungiert im Allgemeinen nicht als grundlegendes Timingmuster, sondern wird vielmehr verwendet, um bestimmte Phrasen rhythmisch besonders hervorzuheben.

\section{Bimetrische Modelle}

In diesem letzteren Beispiel wurde Bimetrik in indirekter Weise mittels extremen Retardierens erzeugt, indem zwei Beat-Unterteilungen simultan vorhanden sind. Auf Basis dieses Modells finden sich sporadisch ganz extreme Verzögerungen und zwar exakt um eine Sechszehntelnote: 


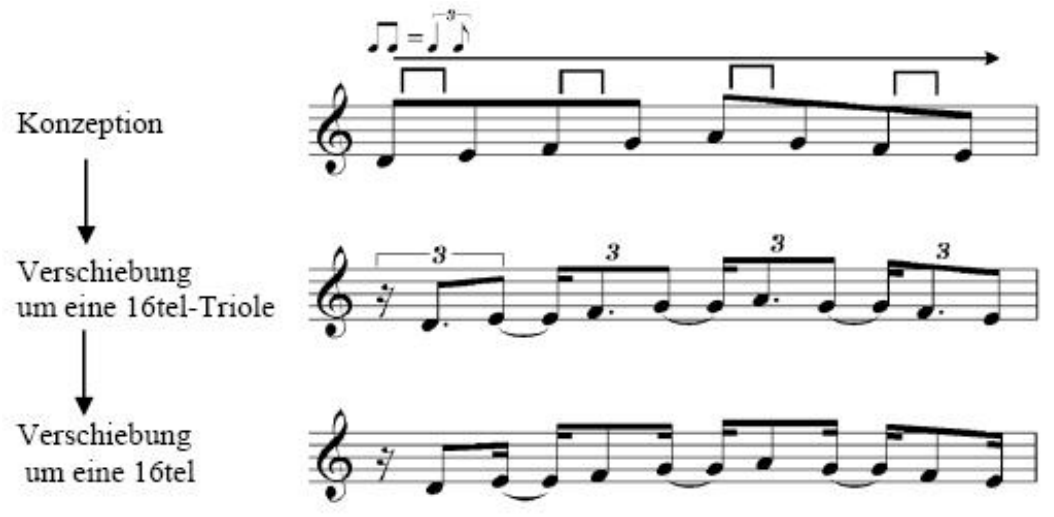

Abb. 14: Exzessive Verschiebung

Bei derart ausgeführten Passagen spielt Scofield in der Regel tenuto:

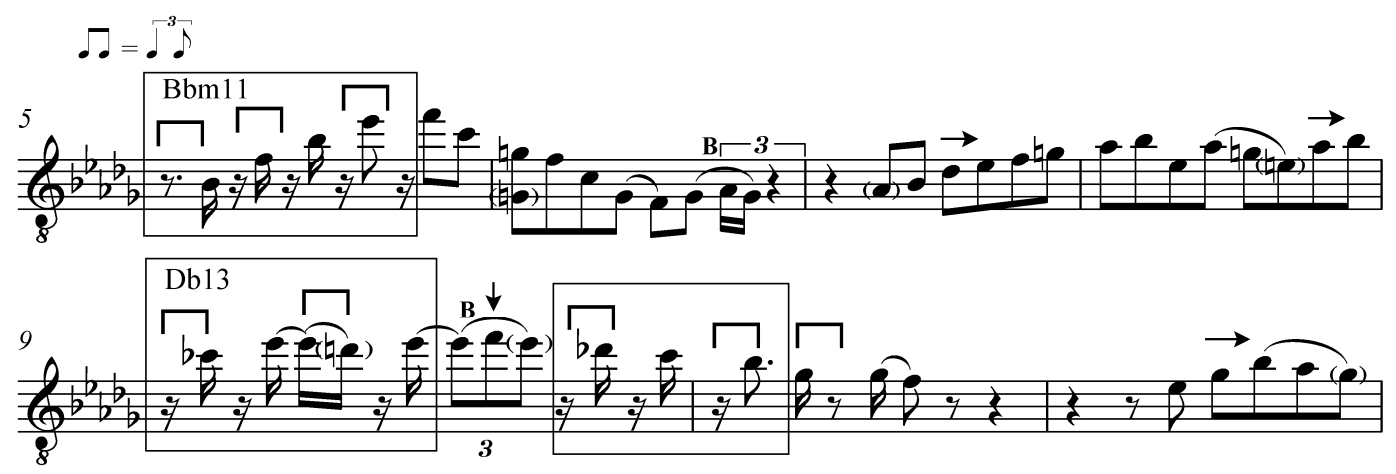

Abb. 15: John Scofield - »|'ll Catch You«, Impr. / A / T. 5-11 (ternär)

Eine grundlegende Technik für Bimetrik-Bildung ist außerdem das DoubleTime- bzw. das Half-Time-Spiel; in diesem Fall in einer Ausführung, in der das Tempo nur vom Soloinstrument verdoppelt oder halbiert wird, nicht jedoch von der Begleitung. Pat Metheny beispielsweise improvisiert im Stück "You Speak My Language« teilweise in Double-Time:

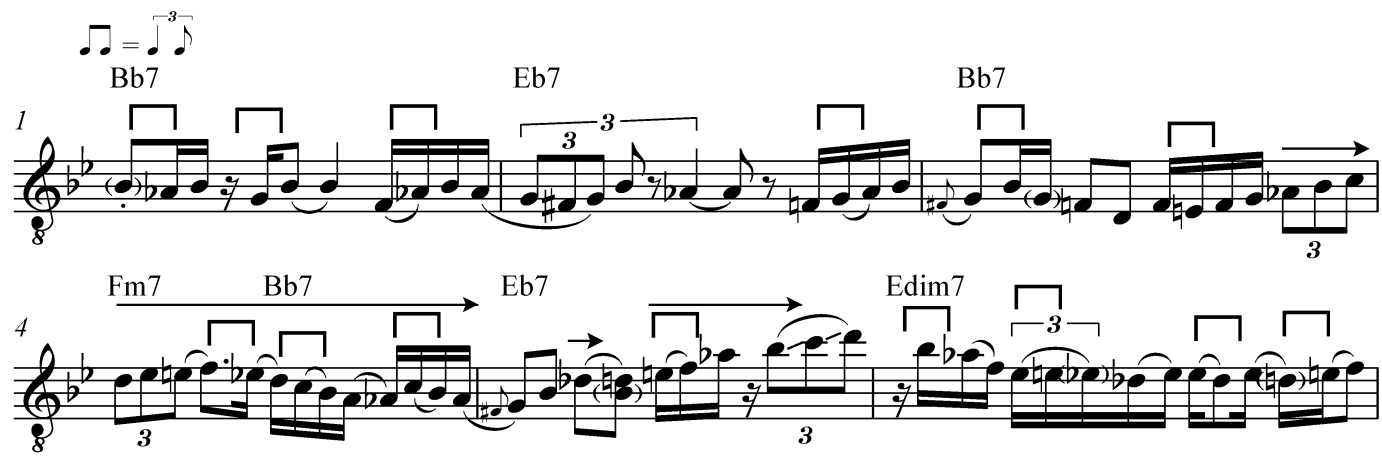

Abb. 16: Pat Metheny - »You Speak My Language«, Impr. 1 / T. 1-6 (ternär) 
Die erste Hälfte des Improvisationschorus von Scofield in »Outta Town « ist durch Half-Time-Spiel charakterisiert:

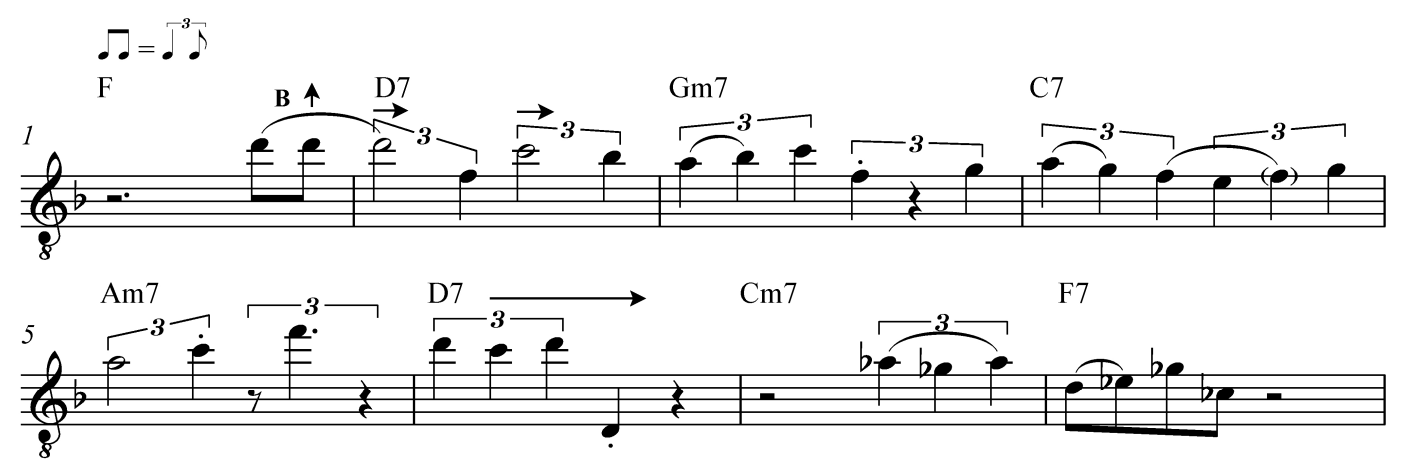

Abb. 17: John Scofield - »Outta Town«, Impr. 1 / A2 / T. 1-8 (ternär)

\section{Weitere Strukturen}

Bis jetzt ging es um Timingstrukturen, die Zählzeit für Zählzeit immer nach demselben Gestaltungsprinzip verlaufen. Es finden sich aber auch Stellen, in denen ein solches kontinuierlich gleichbleibendes Modell nicht zu beobachten ist. Somit ist Timing ein nützliches Mittel zur Variation: Im nächsten Beispielstück »Pat Me « ist das Hauptmerkmal der Themeninterpretation die von Formteil zu Formteil variierte Gestaltung mittels mikrorhythmischer Abweichungen:

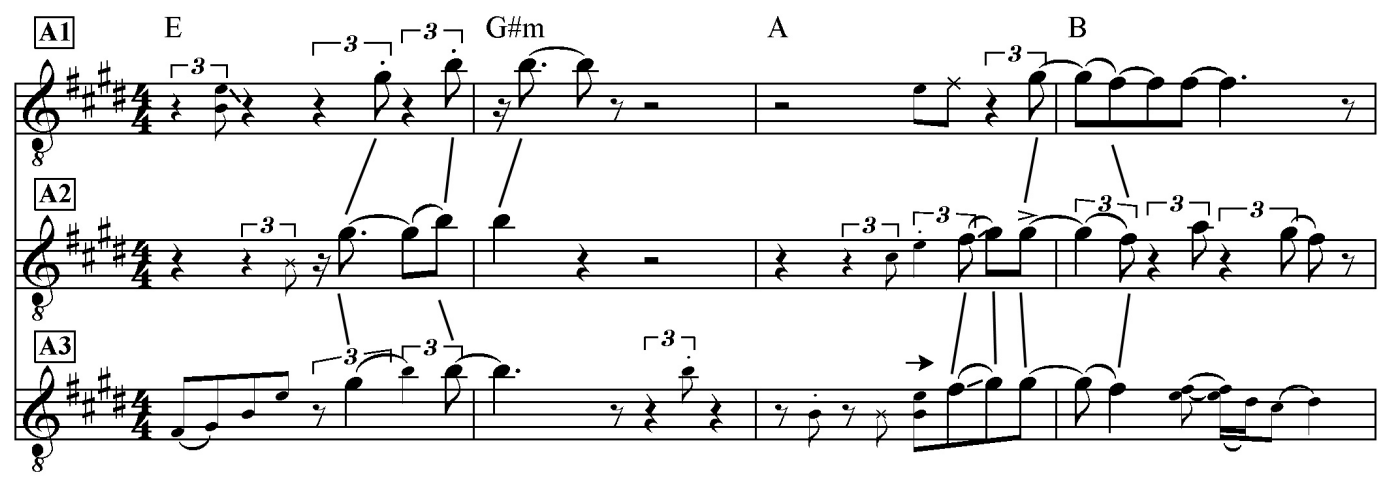

Abb. 18: John Scofield - »Pat Me«, untereinander notierte A-Teile des Themas / T. 1-4 (binär)

Aus dem vorigen Beispiel wird unter anderem auch ersichtlich, dass die Melodiegestaltung durch besonderes Timing eine charakteristische gesangliche Qualität erhält. Hierbei erweckt extremes Retardieren - oft durch makrorhythmische Modifikationen - bei den Zuhörern zumeist ein Gefühl der 
Entspannung. Man kann jedoch ebenfalls rhythmische Irritation und folglich Spannung erzeugen:

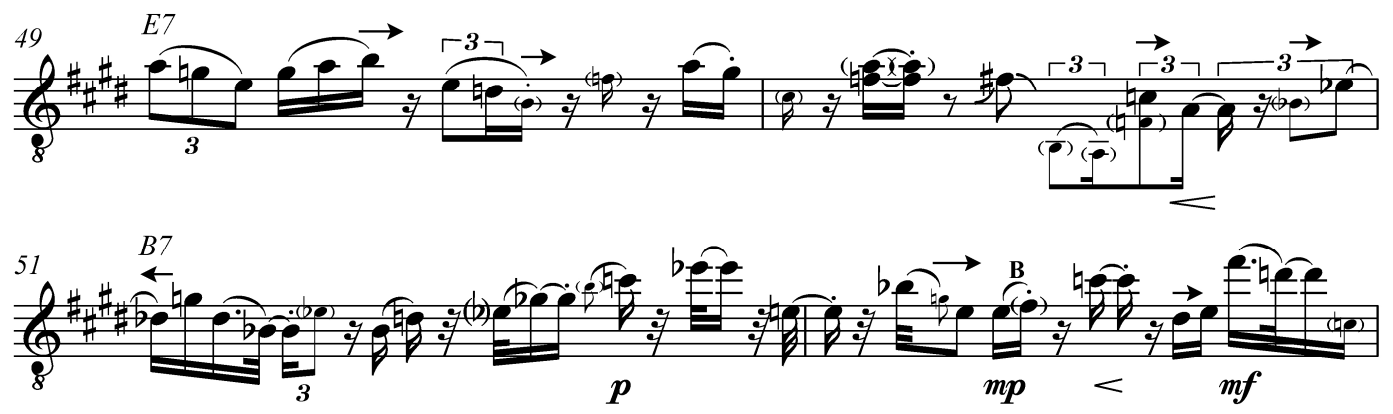

Abb. 19: John Scofield - »Watch Out For Po-Po«, Impr. / T. 49-52 (binär)

\section{Resümee}

Abschließend seien die wichtigsten Anwendungsbereiche der skizzierten Modelle kurz zusammengefasst:

(a) Bestimmte schematische Timingstrukturen spielen in der Genreauthentizität eine fundamentale Rolle (z. B. Swing - Swingphrasierung, Fusion exaktes Timing usw.).

(b) Hinsichtlich der Varianzbildung erweist sich Timing ebenfalls als ein zentraler Faktor. Kontrast kann hauptsächlich erzeugt werden

- durch übliches Variieren im Sinne von Paraphrasieren,

- durch Modifikation des Ausmaßes der rhythmischen Abweichung vom zugrunde liegenden Timingschema (primär von jenem des Swing), womit eine Akzentuierung einer Phrase bewirkt werden kann, sowie

- durch rhythmische Irritation, die im Wesentlichen mittels Bimetrik bzw. hektischer Rubato-Gestaltung erzielt wird.

(c) In interpretatorischer Hinsicht gilt Timing auch als eine der wichtigsten Faktoren für ein kantables Spiel.

Zusammenfassend kann man feststellen, dass Timing einen wesentlichen Einfluss auf den Charakter einer Interpretation hat und bewusst zur Steuerung des Spannungsverlaufes innerhalb eines Stückes eingesetzt werden kann. Das jeweilige Timing eines Interpreten prägt in hohem Maße seinen Personalstil. 


\section{Literatur}

Benadon, Fernando (2006). »Slicing the Beat. Jazz Eight-Notes as Expressive Mikrotiming. «In: Ethnomusicology 50, S. 73-98.

Busse, Walter Gerard (2002). "Toward Objective Measurement and Evaluation of Jazz Piano Performance via MIDI-Based Groove Quantize Templates. «In: Music Perception 19, S. 443-461.

Butterfield, Matthew (2010). »Participatory Discrepancies and the Perception of Beats in Jazz. « In: Music Perception 27, S. 157-176.

Butterfield, Matthew W. (2011). »Why Do Jazz Musicians Swing Their Eighth Notes?« In: Music Theory Spectrum 33, Nr. 1, S. 3-26.

Clarke, Eric F. (1999). »Rhythm and Timing in Music. In: The Psychology of Music. Hg. v. Diana Deutsch. San Diego: Academic Press, S. 473-500.

Collier, Geoffrey L. / Collier, James Lincoln (1996). »Microrhythms in Jazz: A Review of Papers. «In: Annual Review of Jazz Studies 8, S. 117-139.

Collier, Geoffrey L. / Collier, James Lincoln (2002). »A Study of Timing in Two Louis Armstrong Solos. «In: Music Perception 19, S. 463-483.

Collier, Geoffrey L. / Collier, James Lincoln (2007). "Studies of Tempo Using a Double Timing Paradigm. «In: Music Perception 24, S. 229-245.

Ellis, Mark Carlton (1991). »An Analysis of >Swing Subdivision and Asynchronisation in Three Jazz Saxophonists. « In: Perceptual and Motor Skills 73:3, S. 707-713.

Friberg, Anders / Sundström, Andreas (2002). »Swing Ratios and Ensemble Timing in Jazz Performance. Evidence for a Common Rhythmic Pattern. «In: Music Perception 19, S. 333-349.

Goebl, Werner / Palmer, Caroline (2009). "Synchronisation of Timing and Motion Among Performing Musicians. «In: Music Perception 26, S. 427-438.

Honing, Henkjan (2001). »From Time to Time: The Representation of Timing and Tempo.«In: Computer Music Journal 25:3, S. 50-61.

Honing, Henkian / de Haas, W. Bas (2008). "Swing Once More: Relating Timing and Tempo in Expert Jazz Drumming. «In: Music Perception 25, S. 471-476.

Keil, Charles (1995). "The Theory of Participatory Discrepancies: A Progress Report. « In: Ethnomusicology 39, S. 1-19.

Kvifte, Telef (2007). »Categories and Timing: On the Perception of Meter.«In: Ethnomusicology 51:1, S. 64-84.

Marsden, Alan (2007). »Timing in Music and Modal Temporal Logic. In: Journal of Mathematics and Music 1:3, S. 173-189.

Parsons, Will / Cholakis, Ernest (1995). »I Don't Mean a Thang If It Ain't Dang, Danga Dang! «In: Down Beat 62:8, S. 61.

Prögler, J. A. (1995): "Searching for Swing. Participatory Discrepancies in the Jazz Rhythm Section.«In: Ethnomusicology 39, S. 21-54.

Repp, Bruno H. / Windsor, W. Luke / Desain, Peter (2002). »Effects of Tempo on the Timing of Simple Musical Rhythms. «In: Music Perception 19, S. 565-593. 


\title{
Diskographie
}

Johnson, Marc (1987). »Twister. « Auf: Bass Desires: Second Sight, ECM G1351.

Holloway, Ron (1995). »I Found A New Baby. «Auf: Struttin', Milestone Records MCD 9238-2.

McShann, Jay (1978). »Dickie's Dream. « Auf: Big Apple Bash, Atlantic Records KSD 8804.

Metheny, Pat / Scofield, John (1993). "You Speak My Language. A Auf: I Can See Your House From Here, Blue Note CDP 8-27765-2.

Scofield, John (1989). „Nocturnal Mission. « Auf: Time On My Hands, Blue Note B192894.

Scofield, John (1991). »Pat Me.« Auf: Grace Under Pressure, Blue Note CDP 798167-2.

Scofield, John (2000). »'ll Catch You. Auf: Works For Me, Verve 549 281-2.

Scofield, John (2002). »Watch Out For Po-Po.« Auf: Up All Night, Verve 065596.

Stern, Mike (1998). »Outta Town. « Auf: Play, Atlantic Records 7567-93219-2.

Van Ruller, Jesse (2002). »Secret Champ.« Auf: Circles, Criss Cross Jazz 1235.

\begin{abstract}
The term »timing " is a generally used concept in jazz and popular music that neither has a reliable definition, nor has it been described exactly in regards to its implications. In order to define this phenomenon from a stylistic point of view, it can be claimed that this collective term specifies rhythmic particularities, which can be derived from the relation among intended and realised rhythm. The author examines diverse characteristics of timing in the music of contemporary jazz guitarists, illustrated by transcriptions. Improvisations from various styles of music are analyzed and categorized as different types of timing.
\end{abstract}

